Journal of Mathematics and Informatics

Vol. 8, 2017, 25-35

ISSN: 2349-0632 (P), 2349-0640 (online)

Published 14 July 2017

www.researchmathsci.org

DOI: http://dx.doi.org/10.22457/jmi.v8a4

Journal of

Mathematics and

Informatics

\title{
A Study of Recognition about Human Face
}

\author{
Yang Yang ${ }^{1}$, Cong $\mathrm{Yu}^{2}$, Hang $\mathrm{Xiao}^{3}$ and Nan-xiangYu ${ }^{4}$ \\ School of Science, Chongqing University of Posts and Telecommunications \\ Chong'qing-400065, Chongqing, China.

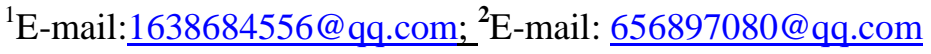 \\ ${ }^{3}$ E-mail:970334034@qq.com; ${ }^{4}$ E-mail:491182600@qq.com
}

Received 6 June 2017; accepted 12 July 2017

\begin{abstract}
This paper studied the simulation problems of facial aging with the computer visual processing software. According to comparing the most used facial pictures, this paper decided to take the face images from FE-NET database as the objects of modeling analysis. Considering the AAM, WT and other Facial Feature Extraction Algorithms, this took the AMM algorithm to extract and mark the facial feature, gray and match histogram. Actually, the high dimensionality of the extracted feature has a bad affection on efficiency and result in post processing, so this paper adopts the classical method PCA to process the facial feature set. This paper adopts a piecewise-linear algorithm to segment all facial images based on ages to four sections, and extract features of every section to make linear similarity compare. This paper simulates images and reverse simulation in every section, with good results.
\end{abstract}

Keywords: PCA algorithm, AAM feature extraction algorithm, piecewise linear algorithm, facial aging model

AMS Mathematics Subject Classification (2010): 68T10

\section{Foreword}

The problem of Human Face Recognition by computer simulating has an important application value so that it become a hot research topic. It has broad prospect of application in police criminal detection, authentication, missing census, military matters of affairs, security and protection, commerce and so on. So it has raised widespread concern by the academic community. The Hidden Markov Model[1] and 3D Model[2] are commonly used to Face Recognition Process. This paper extracts feature, gray color images and match histogram through AAM, and construct a piecewise-linear model by 
Yang Yang, Cong Yu, Hang Xiao and Nanxiang Yu

reducing dimensionality with PCA algorithm, with good results.

\section{Model constructing}

\subsection{Constructing model and flow chart}

(1) To choose testing database and collect face data.

(2) To process the facial images by AAM feature extraction, graying color images, histogram matching.

(3) Reducing dimensionality of the facial feature sets extracted through AAM with PCA[3].

(4) To get simulating results though Piecewise Facial Aging Model.

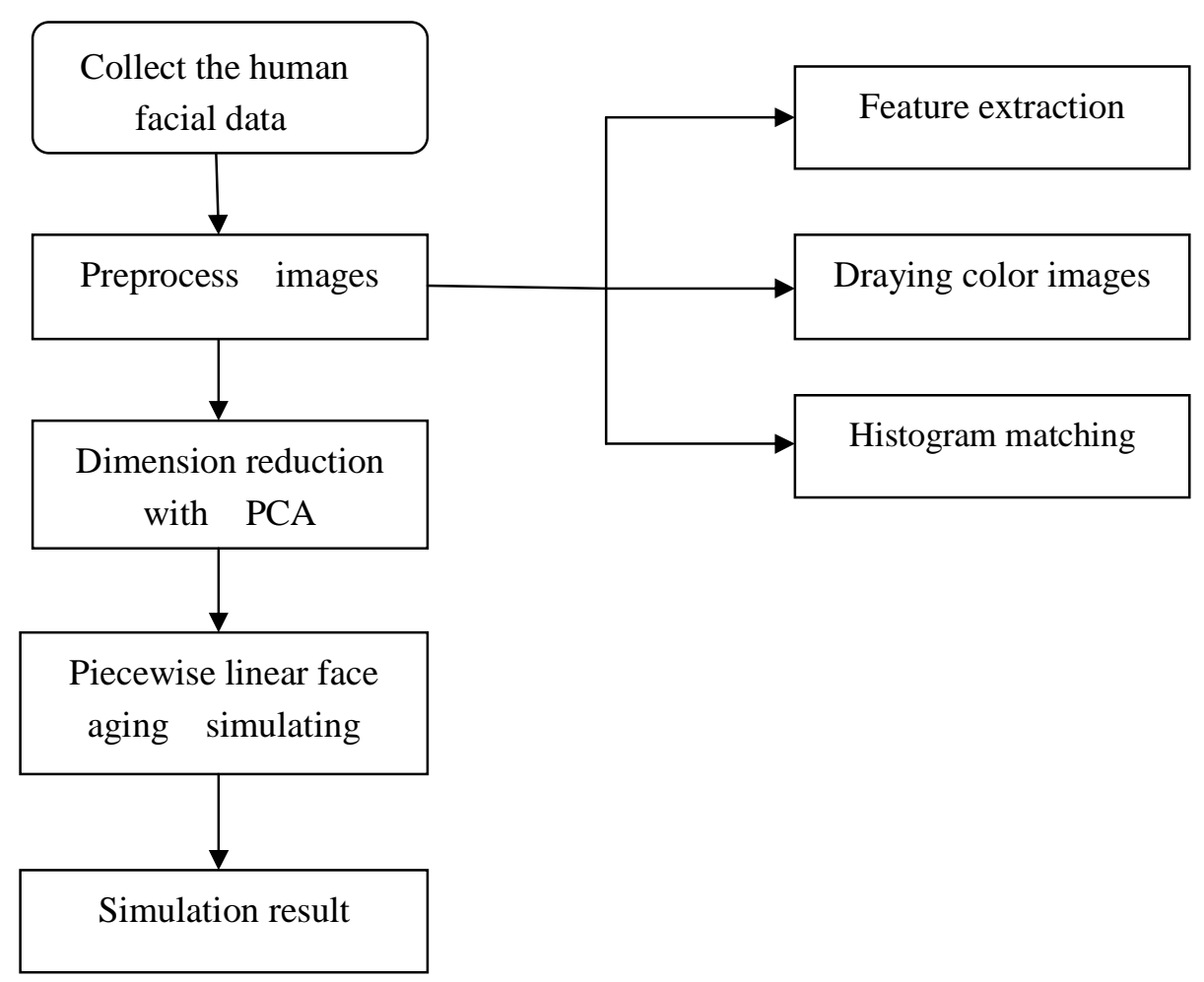

Figure 1: Algorithm flowchart of facial recognition model

\subsection{The choice of testing database and collection of human facial data}

The public database resource of human facial recognition is very scarce as its difficulty to collect enough face pictures of a same person at different ages. According to relative information, there are three databases: MORPH database, FG-NET aging database and FERET database, which are adequate. This paper took the FG-NET database as training 


\section{A Study of Recognition about Human Face}

images data set and to construct model.

FG-NET aging database includes 1002 images about 82 people at the age from 0 to 69 years old (6-18 images of each person). This database provided 68 manual facial features of each image, and other information: size of pic, age, gender, glass, hats, mustache, horizontal and vertical position. As the images are pictured in daily life, the face pictures are affected by the illumination, head position and the facial expressions and so on. However, FE-NET database is the only one available which provides images pictured in ages from 0 to 18 . As following:

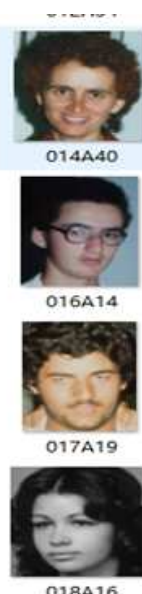

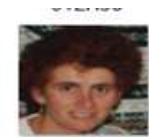
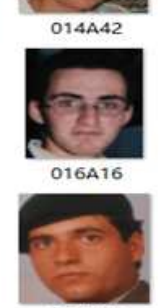

017 A22

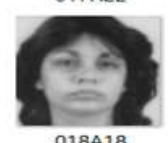

$018 \mathrm{~A} 18$
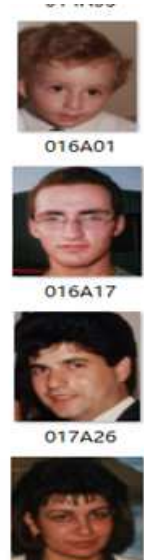

018422
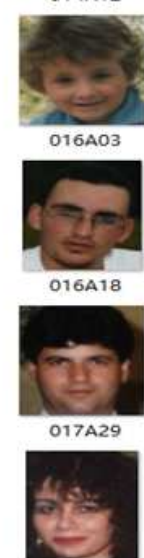

018425

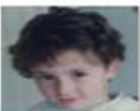

$016 A 04$

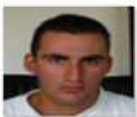

$016 \mathrm{~A} 19$

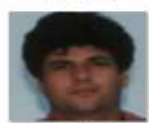

017 A32

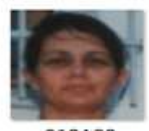

$018 \mathrm{A28}$
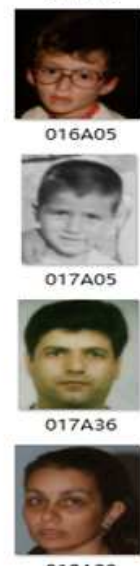

$018 \mathrm{~A} 29$
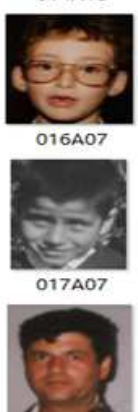

$017 A 38$

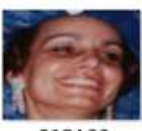

$018 \mathrm{A33}$

Figure 2: The sample images

\subsection{Preprocessing of the human facial picture}

Since the aging process is companied by facial shape and texture. The simulating result would be better based on doing aging training about shape and texture. Therefore, the first step of preprocess is to extract the shape feature that is an effective representation of human face shape and texture features, not related to face shape. Features could be extracted through AAM. Considering the texture features, to gray the color images could reduce the computation cost without affection to the real aging result; Last step, to elimination the affection of illumination to facial texture; To ensure gray uniformed distribution through the histogram matching algorithm[4].

\subsubsection{Constructing of a $\mathrm{AAM}$ and extracting feature information of images}

AAM include modeling construction and corresponding fitting calculation. The first step is to manually flag the manual feature-points on training samples. Then obtain the model expression through the machine learning method, the shape-AAM and texture model expressions. 


\section{Yang Yang, Cong Yu, Hang Xiao and Nanxiang Yu}

(1) Shape modeling

The shape-AAM model implementation steps:

To choose a well training samples;

To mark the manual feature-points on the training samples, s.t the feature-points positions construct a $\mathrm{S}$-shape, denoted by $\mathrm{S}$ and $\mathrm{S}=\left(x_{1}, y_{1}, x_{2}, y_{2}, \ldots \ldots, x_{v}, y_{v}\right)$; denote the number of these positions by $\mathrm{V}$.

To unify the shape through eliminating the global transformation like rotation, scale and translation and so on.

To calculate the eigenvalues and eigenvectors corresponded to the average factors $s_{0}$ and $s_{i}$ through making PCA transform to the unified shape.

To express facial shape by a line ar equation:

$$
\mathrm{S}=S_{0}+\sum_{i=1}^{n} P_{i} S_{i}
$$

(2) Texture modeling construction

Constructing steps of texture-AAM:

Delaunay triangulating the facial shape in the $S_{0}$ and training sets.

Mapping the texture messages of facial shape in the sample to the average shape $S_{0}$ and unifying the texture through the piecewise-linear affine method.

Getting the average texture $A_{0}$, and the eigenvector $A_{i}(\mathrm{i}=1,2, \ldots, \mathrm{m})$ based on unified texture information.

Since the textures are close to shape, facial texture information could be expressed by a linear equation:

$$
\mathrm{A}(\mathrm{X})=(X)+\sum_{i=1}^{n} \lambda_{i} A_{i}(X) \forall X \in S_{0} A_{0}
$$

(3) AAM instance generation

Given parameter $\mathrm{p}$, linearly represented by shape model, then getting a corresponding shape $\mathrm{S}$ and a set of texture data, linearly expressed by a $\lambda$ texture model, a corresponding texture instance $\mathrm{A}(\mathrm{x})$. Then, obtained an AAM instance after mapping the texture information $\mathrm{A}(\mathrm{x})$ of average shape to current shape $\mathrm{S}$.

(4) Fitting calculation

AAM fitting calculation is needed to generating current AAM instance and fitting matching between objects to be determined. Therefore, AAM fitting calculation is the core component of feature-points location in facial recognition. The activity part of AAM model is reflected in the process of fitting calculation, according to the shape modeling, PCA algorithm is well applied to describe the movement of control points. And the shape control points describe the location of the feature points; then, an energy function defined by AAM modeling instance and the sum of squares of the difference from input images: 
A Study of Recognition about Human Face

$$
\sum_{\mathrm{x} \in \mathrm{S}_{0}}\left[\mathrm{~A}_{0}(\mathrm{x})+\sum_{\mathrm{i}=1}^{\mathrm{n}} \lambda_{i} A_{i}(X)-I(W(X, P))\right]^{2}
$$

$\mathrm{I}(\mathrm{W}(\mathrm{X}, \mathrm{P}))$ is the value of the pixel in $\mathrm{W}(\mathrm{X}, \mathrm{P})$ from input image $\mathrm{I}(\mathrm{X})$, it is for estimating the degree of the fitting; In the process of location fitting, the movement of control points could be controlled to generate the current AAM modeling instance according the linear expression.

While judging the shape parameter $\mathrm{P}$ and the value of texture parameter the shape control points is controlled, then an AAM instance generated, keep iterative repetition to minimize the energy function, as well as the modeling instance and the input image are achieved. Finally, the shape controlled points described the feature points of current object.

AAM feature extraction method considered the partial feature information but also the global shape and texture information. It can realize velocity and feature posting accurate. In conclusion, this paper adopted features extraction method based on AAM.

\subsubsection{Graying color images}

The process of graying color image is called graying process. Every pixel in color image is made up of three components, the R, G, B component. every component is graded to 255 different values, so there are more than 16 million colors of every component. While the three components $\mathrm{R}, \mathrm{G}, \mathrm{B}$ are the same value, then grayscale image. Since the variation range of every pixel is from 0 to 255 , the common practice is to convert initial pictures to grayscale image to reduce the computation cost. As the color image, to describe the features of a grayscale image also present the global and partial distribution feature of color and brightness levels.

While $\mathrm{R}=\mathrm{G}=\mathrm{B}$, the color is gray color, the value of any component is gray value. So only one byte is needed to storage single the information of single pixel. This paper takes the mean value method to graying image, to obtain mean value by simple average operation to the R, G, B components. Then, grayscale image is obtained.

\subsubsection{Histogram matching}

The last step of preprocessing is to histogram matching, which could eliminate the luminance difference in the face database caused by illumination.

The step of histogram matching method:

Given initial image $P_{r}(r)$ and the gray distribution probability of target image $P_{z}(z)$, histogram matching is to judge the histogram to make it like the shape of $P_{z}(z)$, deniote S: 
Yang Yang, Cong Yu, Hang Xiao and Nanxiang Yu

$$
\mathrm{s}=\mathrm{T}(\mathrm{r})=\int_{0}^{r} P_{r}(w) d w
$$

$\mathrm{W}$ is the variable of integration, define a random variable $\mathrm{Z}$ :

$$
\mathrm{G}(\mathrm{z})=\int_{0}^{z} p_{z}(t) d t=s
$$

$\mathrm{t}$ is a random variable. Then the equation of $\mathrm{Z}$ is:

$$
\mathrm{z}=G^{-1}(s)=G^{-1}[T(r)]
$$

Then, the transform function is obtained, assuming that there exists a function $G^{-1}$, s.t:

(1) $G^{-1}$ is monotonic increasing in the section $0<s<1$;

(2) $0<G^{-1}<1$, while $0<\mathrm{s}<1$

Through the three steps of processing, the facial images are well to mode constructing, the following picture is processed:
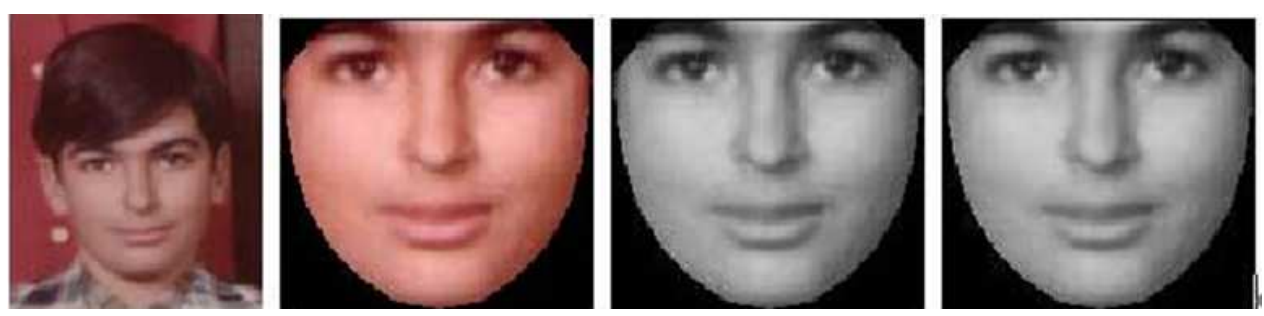

Figure 3: The processed result based on the testing image

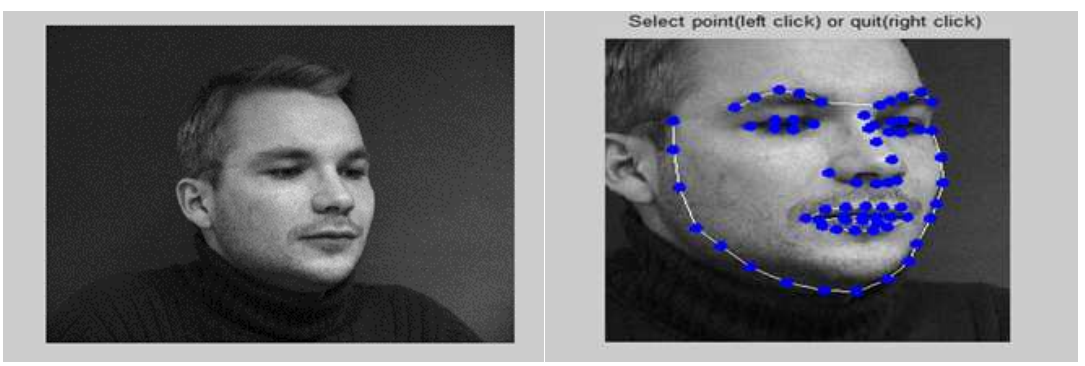

Figure 4: Marked result through AAM algorithm

\subsection{The model constructing based on the PCA algorithm}

\subsubsection{Introduction of PCA theory}

$\mathrm{X}$ is a m-dimensional random variable, assuming the mean value of $\mathrm{X}$ is 0 , then:

$$
E(X)=0
$$

$\mathrm{W}$ is a m-dimensional variable unit vector. Scalar product of Vector $\mathrm{X}$ and $\mathrm{w}$ is expressed 
A Study of Recognition about Human Face

by:

$$
\begin{gathered}
y=\sum_{k=1}^{n} w_{k} x_{k}=w^{T} x \\
\text { s.t }|| \mathrm{w}||=\left(\mathrm{w}^{\mathrm{T}} \mathrm{w}\right)^{1 / 2}=1
\end{gathered}
$$

the target of PCA is to figure out weight vector $w$, to maximize the expression $E\left(y^{2}\right)$ :

$$
E\left[y^{2}\right]=E\left[\left(w^{T} x\right)^{2}\right]=w^{T} E\left[x x^{T}\right] w=w^{T} C_{x} w
$$

According to the linear algebra theory, the target value of $w$ provides that:

$$
C_{x} w_{j}=\lambda_{j} w_{j}
$$

The target value of $\mathrm{w}$ is the eigenvector of the biggest eigenvalue of matrix $C_{x}$

\subsubsection{PCA theory algorithm implementation}

Steps of algorithm:

Supposing the sample set of observed value is a n-dimensional random vector whose expectation value is 0 (If not, do $\mathrm{DE}$ averaging preprocess, which means doing a translation of transformation): $\left\{\mathrm{x}_{\mathrm{i}} \in \mathrm{R}^{\mathrm{n}} ; \mathrm{j}=1,2, \ldots, \mathrm{l} ; \sum_{\mathrm{j}=1}^{\mathrm{l}} \mathrm{x}_{\mathrm{i}}=0\right\}$, and to take the covariance matrix as source matrix.

$\mu$ is the mean vector of observed sample, as $x_{t}$ is a zero vector. So the value of $\mu$ is 0 . Naturally, matrix $\sum$ is a $n \times n$ symmetric and positive semi-definite matrix, doing eigenvalue decomposition, then:

$$
U^{T} \sum U=\left[\begin{array}{cccc}
\lambda_{1} & 0 & \cdots & 0 \\
0 & \lambda_{2} & \cdots & 0 \\
\vdots & \vdots & \cdots & \vdots \\
0 & 0 & \cdots & \lambda_{n}
\end{array}\right]
$$

$\mathrm{U}$ is a orthogonal matrix, $\lambda_{\mathrm{i}}(\mathrm{i}=1,2 \ldots, \mathrm{n})$ is the eigenvalue of matrix $\sum$, and $\lambda_{1} \geq \lambda_{2} \geq \cdots \geq \lambda_{\mathrm{n}} \geq 0$, the eigenvector is every single column of matrix $\mathrm{U}(\mathrm{L}$ to $\mathrm{R})$, they are a set of orthogonal basis.

A linear transformation: $y=u^{T} x$, the covariance matrix of the eigenvector $\left\{y_{j} \in R^{n} ; j=\right.$ $\left.1,2, \ldots, \mathrm{l} ; \sum_{\mathrm{j}=1}^{\mathrm{l}} \mathrm{j}=0\right\}$ is:

$$
E\left(y y^{T}\right)=E\left(U^{T} x x^{T} U\right)=U^{T} \sum U=\left[\begin{array}{cccc}
\lambda_{1} & 0 & \cdots & 0 \\
0 & \lambda_{2} & \cdots & 0 \\
\vdots & \vdots & \cdots & \vdots \\
0 & 0 & \cdots & \lambda_{n}
\end{array}\right]
$$

The $\mathrm{y}_{\mathrm{i}}(\mathrm{i}=1,2, \ldots \mathrm{n})=\mathrm{L}$ in the new eigenvector is called the ith primal component. 


\section{Yang Yang, Cong Yu, Hang Xiao and Nanxiang Yu}

Obviously, the ith eigenvalue of $\mathrm{t} \sum$ equals to the variance $\lambda_{i}$, the variance contribution rate of ith primal component is:

$$
\frac{\lambda_{i}}{\sum_{i=1}^{l} \lambda_{i}}
$$

The sum of first $m$ primal component variance contribution rate is:

$$
\frac{\sum_{i=1}^{m} \lambda_{i}}{\sum_{i=1}^{l} \lambda_{i}}
$$

While the first $\mathrm{m}$ primal component is a big enough one, we can take the $\mathrm{m}$ primal component as the new features, truncate the other features. So the extraction of data sample is done, then the minimize value of the reconstruct variance is:

$$
\min \mathrm{J}_{\mathrm{e}}=\sum_{\mathrm{i}=\mathrm{m}+1}^{1} \lambda_{i}
$$

\subsection{Model constructing and solution algorithm based on piecewise linear aging process}

Taking the process of facial aging as the study object, to simulate the aging process piecewise-linearly on all age sections according to the facial hierarchical structure model based on the process above all.

\subsubsection{Model constructing of piecewise aging process}

The global prototype represents the general shape and texture feature of facial pictures in all ages. And it could not work well for high frequency information characteristics, like wrinkles. Considering the similarity principle, to simulate the aging similarity along to the facial aging process. The global prototype is a set of average human facial picture, making a mapping from similar human face matching to the aging process.

The detailed procedures of the algorithm:

Presenting the initial face picture and extract the shape and texture massage based on hierarchical structure[5].

Getting aging simulate result in every adjacent age section according the extracted feature information simulated with global prototype.

Doing similar matching in every age group, which means to find the most similar face picture in age groups and to search the most similar face picture.

Calculating the variance between two images and map to original picture, taking the aging simulating result and retrosynthetic the aging simulating result according the hierarchical structure.

Return to the first step, while aging simulating result doesn't equal to the objective result.

There are four stages of the whole process of aging, including childhood, youth, 


\section{A Study of Recognition about Human Face}

middle age, old age. The changing process between adjacent stages is gradual rather than a skip from last stage to the next one. Naturally, the aging simulating process is the same about the natural law. If the age of face in the initial images is not close to the target one, first to do a simulating in the adjacent age group, then further aging simulating until the target age group to synthesis the final result. The process of aging simulating could be expressed detailedly with more detailed data, choose initial facial images from the four stages and extract the shape and texture feature, then obtain the basis expression through PCA algorithm and plotted in 3-D space. If the change of face shape and texture vector along with ages is linear, the change trend of 3-dimensionlity vector of reduced-dimension transformation should also be linear. However, the result in Pic3 doesn't show a linear change trend of the shape and texture vector in the 3-D space. Therefore, the process of facial aging is not a direct linear process.

To do a piecewise linear aging simulating about integral layer and detail layer[6], the process of aging simulating is to do the global piecewise-aging simulating on integral layer and the local facial component and winkles in the detailed layer, then resynthesize the aging result according to the hierarchical structure.

\subsubsection{Solution algorithm based on the piecewise aging process}

Doing global aging simulating to the new face picture in first integral layer by facial global prototype and making aging simulating with global shape and texture respectively, then aligning the shape aging result to the texture result.

To eliminate the face position in picture and the affection of accuracy caused by angle. Unifying the new face picture shape vector and the shape vector is necessary. Then, the aging process was not linear and to do aging simulating on the new face picture shape to the adjacent age group until the target ages. The average shape of every age group in global prototype described the shape feature of current age group.

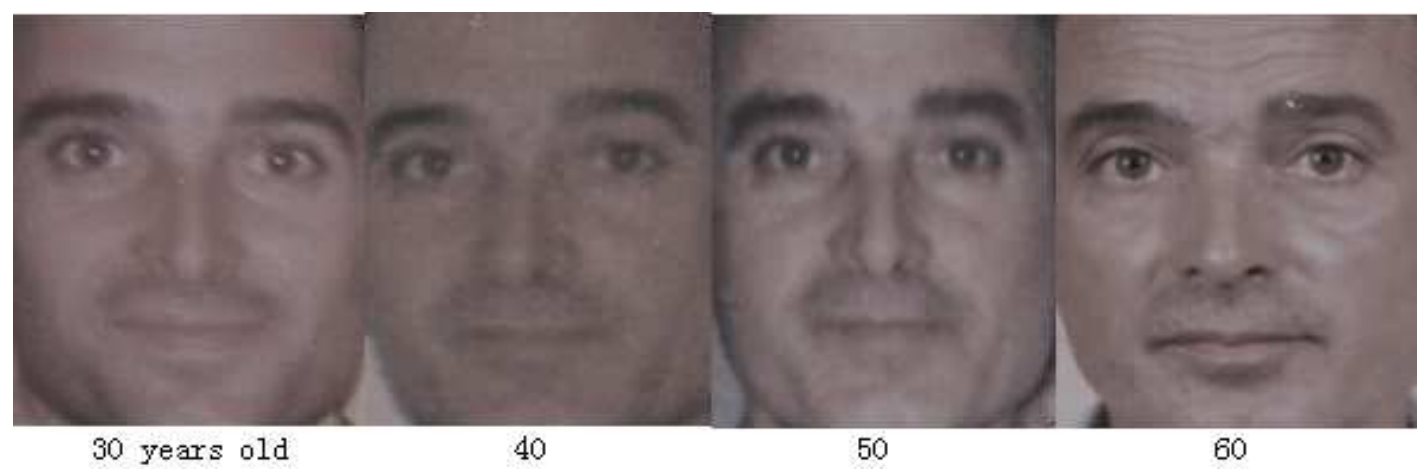

Figure 5: The simulated change of face from 30 to 60 
Yang Yang, Cong Yu, Hang Xiao and Nanxiang Yu

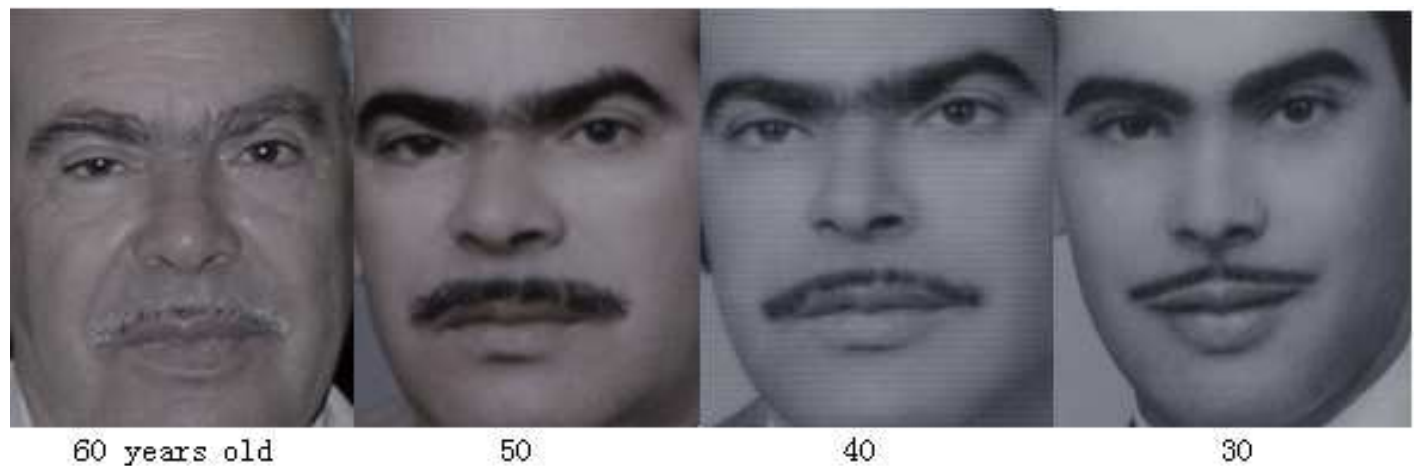

Figure 6: Reverse simulating the change from 60 to 30

\subsection{Analyze about the result}

We analyzed the change of face from the 30 to 60 years old and reverse it. With good results according to the compare with the original images.

\section{Conclusion}

This paper marked and extracted facial feature and gray the pictures, histogram matching according AAM model with a higher efficiency based on the relative paper.

Then, reducing dimensionality of facial feature extracted with AAM through PCA makes the facial information more representative. And the image simulating of facial recognition based on piecewise-linear algorithm got a good result. It provides a scientific basis for relative study.

Acknowledgement. This work was supported in part by the Education and teaching reform fund of Chongqing University of Posts and Telecommunications under Grant XJG1636.

\section{REFERENCES}

1. A.V.Nefian, A Hidden Markov Model-Based Approach for Face Detection and Recognition, $\mathrm{PhD}$ dissertation in Electrical Engineering, Georgia Institute of Technology, (1999).

2. P.Paysan, R.Knothe, B.Amberg, et al., A 3D face model for pose and illumination invariant face recognition, IEEE International Conference on Advanced Video and Signal Based Surveillance, (2009) pp.296-301.

3. R.Gottumukkal and V.K.Asari, Letters An improved face recongnition technique based on modular PCA approach, Pattern Recognition, 25(3) (2004) 429-436.

4. A.M.Sevcenco and W.S.Lu, Perfect histogram matching PCA for face recognition, 
A Study of Recognition about Human Face

Kluwer Academic Publishers, (2010).

5. F.Samaria and S.Young, HMM-based architecture for face identification, Image \& Vision Computing, 12(8) (1994) 537-543.

6. D.C.Hood, B.Fortune, M.A.Mavrommatis, et al., Details of glaucomatous damage are better seen on OCT en face images than on OCT retinal nerve fiber layer thickness maps, Investigative Ophthalmology \& Visual Science, 56(11) (2015) 6208-6216. 